\title{
Effects of dietary acetaminophen and vitamin $C$ supplement on serum cortisol and tumor necrosis factor-alpha concentrations in pigs vaccinated with foot-and-mouth disease vaccine
}

\author{
Chun-Nam Cha ${ }^{1, \dagger}$, Beom-Jun Lee ${ }^{2, \uparrow}$, Eun-Kee Park ${ }^{3}$, Chang-Yeol Yoo ${ }^{4}$, Suk Kim ${ }^{5}$, Hu-Jang Lee ${ }^{5, *}$ \\ ${ }^{1}$ Engineering Research Institute and Department of Industrial Systems Engineering, and ${ }^{5}$ College of Veterinary Medicine and \\ Institute of Animal Medicine, Gyeongsang National University, Jinju 52828, Korea \\ ${ }^{2}$ College of Veterinary Medicine and Research Institute of Veterinary Medicine, Chungbuk National University, \\ Cheongju 28644, Korea \\ ${ }^{3}$ Department of Medical Humanities and Social Medicine, College of Medicine, Kosin University, Busan 49267, Korea \\ ${ }^{4}$ Department of Computer Information, Gyeongnam Provincial Namhae College, Namhae 52422, Korea
}

(Received: April 5, 2017; Revised: June 13, 2017; Accepted: July 14, 2017)

\begin{abstract}
This study evaluated the effect of a combination of acetaminophen and vitamin C (CAV) on reducing serum cortisol and tumor necrosis factor- $\alpha$ (TNF- $\alpha$ ) concentrations in piglets vaccinated with foot-and-mouth disease (FMD) vaccine. Piglets were vaccinated with FMD vaccine and treated with $\mathrm{CAV}$ at concentrations of $0.0,0.5,1.0$, and 2.0 $\mathrm{kg} /$ ton feed (P-CON, AD-1, AD-2, and AD-3, groups, respectively) for 5 days post-vaccination. Cortisol and TNF- $\alpha$ levels at 5 days post-treatment in the AD-1-3 groups were significantly lower than that in the P-CON group $(p<0.05)$. There were no significant differences between AD-2 and AD-3 groups and non-vaccinated, non-CAV-treated piglets.
\end{abstract}

Keywords: acetaminophen, cortisol, foot-and-mouse disease vaccination, pigs, tumor necrosis factor-alpha

Foot-and-mouth disease (FMD) is recognized as one of the most contagious viral diseases that affects cloven-hoofed animals such as cattle, pig, sheep and goat. FMD causes severe economic loss and is characterized by high morbidity, low mortality and the development of vesicles and erosions in the mucosa of the mouth and skin of the interdigital spaces and coronary band [7]. Foot-and-mouth disease virus (FMDV) belongs to the genus Aphthovirus in the family Picornaviridae and has seven types, O, A, Asia1, C, and the three South African Territories (SAT) types 1-3 [5].

Up to now, several FMD outbreaks occurred in the Republic of Korea in 2000, 2002, 2010-2011 and 2014-2015 [7, 9]. Since the FMD outbreak of 2010-2011, a national blanket vaccination policy has been enforced for ruminants and pigs as a sustainable preventive measure, and a routine vaccination program is still a central control tool against FMDV infection, although this program is somewhat controversial [9].

Considering FMD outbreaks in surrounding countries, typical vaccinations were started in September 2011 in the Republic of Korea using trivalent FMD vaccines (types O, A, and Asia1) [7]. The trivalent FMD vaccine consists of inactivated viruses with double oil-based emulsion and includes structural proteins of FMD viruses (O1 Manisa + A Malaysia + Asia 1 Shamir serotypes) [9]. Although multivalent FMD vaccines reduced the large FMD outbreak, a single-dose injection of the trivalent FMD vaccine in pigs has many problems, including poor humoral immune induction and a short period of immune duration compared to that induced by natural FMDV infection [9]. Also, the vaccination practice can be a stressful management practice to the animal due to the act of handling of the animals, the injection, or a possible inflammatory response, and some adverse effects caused by the use of vaccination have been reported [15].

Immune responses of animals to FMD vaccines have been extensively investigated [8]. However, there are few published reports on the control of stress and inflammatory responses in pigs after FMD vaccination. Therefore, the present study evaluated the reductive potential of a combination of acetaminophen and vitamin C (CAV) on stress and inflammatory responses in pigs post-FMD vaccination.

The drug (AD-Anyless Powder) was purchased from AD Biotech (Korea). This medication contains $20 \%$ of acetaminophen and $10 \%$ of vitamin $\mathrm{C}$. The study was conducted on a livestock farm located in near the Jinju area in Gyeong-

\footnotetext{
*Corresponding author

Tel: +82-55-772-2352, Fax: +82-55-772-2308

E-mail: hujang@gnu.ac.kr

The first two authors contributed equally to this work.
} 
sangnam-do Province, Korea. Twenty five 12-week-old pigs $[($ Duroc $\times$ Yorkshire $) \times$ Landrace $]$ without any history of FMD vaccination were selected and randomly divided into 5 groups of 5 pigs. Five experimental groups consisted of negative control $(\mathrm{N}-\mathrm{CON})$, positive control $(\mathrm{P}-\mathrm{CON})$ and three drug treatment groups (AD-1, AD-2 and AD-3). The N-CON was a non-vaccinated group and administered with the normal feed. Also, P-CON, AD-1, AD-2 and AD-3 were vaccinated with FMD vaccine and administered with the drug at the concentration of $0.0,0.5,1.0$ and $2.0 \mathrm{~kg}$ per ton feed, respectively, for consecutive 5 days post-vaccination. The FMD vaccine was an inactivated trivalent FMDV vaccine (PROVAC FMD) purchased from Komipharm International (Korea). This vaccine formulated a double oil-based emulsion adjuvant with at least six $50 \%$ protective doses of inactivated trivalent FMDVs (O1 Manisa + A Malaysia + Asia 1 Shamir serotypes). All animal experiments were conducted under ethics approval from the Gyeongsang National University Animal Ethics Committee in accordance with the guidelines of the Korean Council on Animal Care (GNU-160831-M0052).

On the 1st, 3rd and 5th days after administration of the drug, blood samples were collected into a heparinized vacutainer tube from the jugular vein of pigs. All blood samples were centrifuged at 2,000 $\times \mathrm{g}$ for $10 \mathrm{~min}$ to separate the serum. According to the manual procedures, cortisol and tumor necrosis factor-alpha (TNF- $\alpha$ ) concentrations in the serum were analyzed with the bovine cortisol enzyme-linked immunosorbent assay (ELISA) kit and TNF- $\alpha$ ELISA kit purchased from Cusabio Biotech (USA), respectively.

All results were represented as the mean \pm SD. Statistical analyses were performed using SPSS software (ver. 18.0; SPSS, USA). Data were analyzed using one-way analysis of variance (ANOVA), followed by student's two-tailed $t$-test. The value of $p<0.05$ was used as the criterion for statistical significance.

Changes in serum cortisol concentrations were presented in Figure 1. On day 1 after treatment, three were no significant differences in serum cortisol concentrations between N-CON and all groups vaccinated with FMD vaccine. On day 3 posttreatment, cortisol levels in all FMD vaccinated groups were increased. Also, cortisol concentrations in AD-1 and AD-2 were significatly increased compared to those in N-CON, but were not significatly different compared to those in P-CON. However, cortisol level in AD-3 was significantly decreased compared to that in P-CON and not significant different compared to that in N-CON. On day 5 post-treatment, cortisol levels in all drug treatment groups were significantly decreased compared to that in P-CON, and cortisol concentrations in AD-2 and AD-3 were not significatly different compared to those in N-CON. In the present study, the drug $(1.0 \mathrm{~kg} / \mathrm{ton}$ feed) showed the potential effect of the reduction of the FMD vaccine-stress in pigs after treatment for 5 days.

The FMD vaccines that are currently used worldwide only consist of inactivated vaccines which are commonly produced with as gel- or oil-adjuvant depending on the serotype

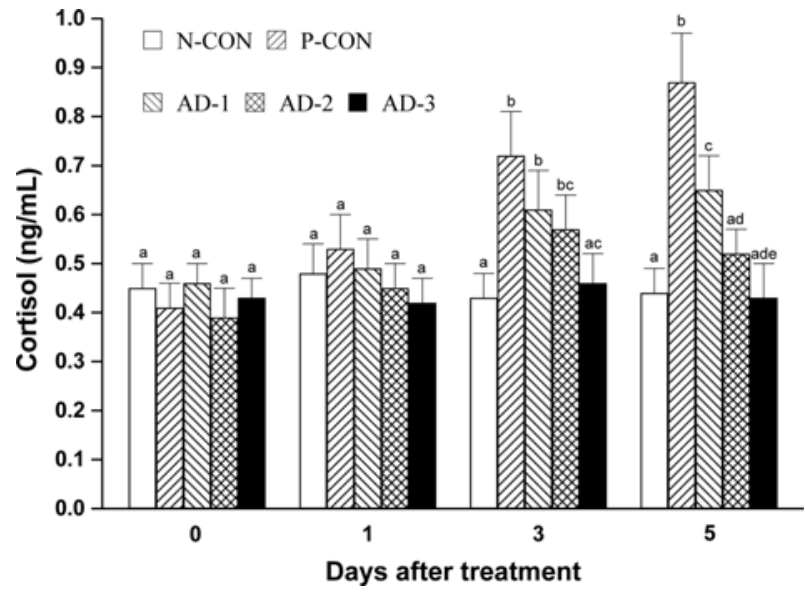

Fig. 1. Change of cortisol concentrations in serum of piglets. N$\mathrm{CON}$, administered with normal feed; P-CON, administered with normal feed with FMD vaccination; AD-1, administered with the drug at the dose of $0.5 \mathrm{~kg} /$ ton feed for 5 days postFMD vaccination; $\mathrm{AD}-2$, administered with the drug at the dose of $1.0 \mathrm{~kg} / \mathrm{ton}$ feed for 5 days post-FMD vaccination; AD-3, administered with the drug at the dose of $2.0 \mathrm{~kg} /$ ton feed for 5 days post-FMD vaccination. Different superscripts mean significantly different $(p<0.05)$ within the same day after treatment.

[9]. In Korea, oil-adjuvanted vaccines against three serotypes, O (O1 Manisa), A (A Malaysia 97), and Asia 1 (Asia 1 Shamir), have continued to be vaccinated since early 2011 [7]. According to the results of a survey on animal responses after such FMD inoculation practices, stress, fever, pain, loss of appetite, lethargy, and temporary decrease in milk production and growth rate in some cases were observed among the vaccinated animals [7].

In pigs, many conditions have been considered to be stressors such as vaccination, weaning, mixing and transportation and have been shown to lead to increased blood concentrations of cortisol [1, 3]. Acetaminophen is an analgesic and antipyretic agent and the antinociceptive effect of the drug has been reported in animal models of pain [4]. Van Der Staay et al. [13] reported that salivary cortisol concentration in piglets subcutaneously injected with butafosfan $(20 \mathrm{mg} / \mathrm{kg}$ body weight $[\mathrm{BW}]$ ) was reduced during the first $2 \mathrm{~h}$ of the encounter with a pig from a different litter. In addition, cortisol level of piglets treated with ketoprofen $(3 \mathrm{mg} / \mathrm{kg} \mathrm{BW})$ before castration was significantly reduced at $90 \mathrm{~min}$ postcastration compared with that of non-treated piglets $(p<0.05)$ [2]. Furthermore, piglets receiving meloxicam and flunixin had significantly lower values of serum cortisol at $4 \mathrm{~h}$ after castration than the control group [6, 10]. Considering the dosage, route and period of administration, the CAV at the concentration of 1.0 and $2.0 \mathrm{~kg} /$ ton feed in this study was more efficient and long-lasting than above analgesics such as butafosfan, ketoprofen, meloxicam and flunixin in reducing the stress of FMD vaccination.

Changes of serum TNF- $\alpha$ concentrations were presented in 
Table 1. Changes of tumor necrosis factor (TNF)- $\alpha$ concentration in serum of piglets after drug treatment

\begin{tabular}{lcccc}
\hline \hline \multirow{2}{*}{ Groups } & \multicolumn{4}{c}{ Days after treatment } \\
\cline { 2 - 5 } & 0 & 1 & 3 & 5 \\
\hline N-CON & $225.81 \pm 9.35^{\mathrm{a}}$ & $223.47 \pm 10.17^{\mathrm{a}}$ & $225.73 \pm 9.52^{\mathrm{a}}$ & $224.52 \pm 9.91^{\mathrm{a}}$ \\
P-CON & $223.48 \pm 11.36^{\mathrm{a}}$ & $263.63 \pm 12.51^{\mathrm{b}}$ & $271.25 \pm 14.29^{\mathrm{b}}$ & $278.41 \pm 15.83^{\mathrm{b}}$ \\
AD-1 & $221.65 \pm 12.54^{\mathrm{a}}$ & $254.58 \pm 11.89^{\mathrm{b}}$ & $266.32 \pm 12.71^{\mathrm{c}}$ & $249.22 \pm 13.52^{\mathrm{c}}$ \\
AD-2 & $224.51 \pm 10.65^{\mathrm{a}}$ & $247.62 \pm 13.73^{\mathrm{b}}$ & $251.38 \pm 11.54^{\mathrm{a}}$ & $235.49 \pm 12.37^{\mathrm{ac}}$ \\
AD-3 & $225.83 \pm 13.92^{\mathrm{a}}$ & $239.47 \pm 14.68^{\mathrm{ab}}$ & $235.74 \pm 10.46^{\mathrm{a}}$ & $227.51 \pm 11.96^{\mathrm{ac}}$ \\
\hline
\end{tabular}

Different superscripts within the same column mean significantly different $(p<0.05)$.

Table 1. On day 1 post-treatment, TNF- $\alpha$ concentrations in $\mathrm{AD}-1$ and $\mathrm{AD}-2$ were significantly different compared to those in N-CON, but TNF- $\alpha$ level in AD-3 was not significantly different compared to that in N-CON and P-CON. On day 3 after treatment, TNF- $\alpha$ concentration in AD-1 was significatly decreased compared to that in P-CON, but significatly increased compared to that in N-CON. However, TNF$\alpha$ level in AD-2 and AD-3 were significantly decreased compared to that in $\mathrm{P}-\mathrm{CON}$, and not significantly different compared to that in N-CON. On day 5 post-treatment, TNF- $\alpha$ levels in all drug treatment groups were significantly decreaed compared to those in P-CON, but not significantly diffent compared to those in $\mathrm{N}-\mathrm{CON}$.

Adjuvants of FMD vaccines elicit innate immune responses associated with antigen presentation to T-cell lymphocytes, including inflammatory and acute-phase reactions [11]. In a previous study [8], TNF- $\alpha$ level was decreased in FMD vaccinated-pigs supplemented with germanium biotite $(30 \mathrm{~kg} / \mathrm{ton}$ feed) for 5 weeks, but there were no significant differences between the treatment pigs and control. In another previous study [14], $\beta$-glucan (15 mg/kg BW/day) was orally administered to piglets for 14 days following lipopolysaccharide (LPS) challenge. Results of the study showed that there was significantly different in TNF- $\alpha$ concentration between $\beta$-glucantreated group and non-treated group at $3 \mathrm{~h}$ after LPS challenge. Compared with above previous studies, the CAV at the concentration of 1.0 and $2.0 \mathrm{~kg} /$ ton feed was more effective than germanium biotite and less effective than $\beta$-glucan in the decrease of serum TNF- $\alpha$ level. In this study, the CAV has an effect on the reduction of serum TNF- $\alpha$ concentration because vitamin $\mathrm{C}$ contained in the $\mathrm{CAV}$ inhibits nuclear transcription factor kappa $\mathrm{B}$, which is responsible for the activation of some pro-inflammatory cytokines such as TNF$\alpha$, interleukin (IL)-1, IL-6 and IL-8 [12].

In the present study, the efficacy of AD-2 and AD-3 against stress and inflammatory responses caused by FMD vaccination was demonstrated by improving the symptoms caused by FMD vaccination, decreasing serum cortisol and TNF- $\alpha$ concentration. Results from this study suggest that dietary supplement of the CAV at the level of $1.0 \mathrm{~kg} /$ ton feed for 5 days after FMD vaccination may be useful for the release of stress and inflammation by FMD vaccination.

\section{Acknowledgments}

This research was supported by Dae Han New Pharm Co. Ltd. (Seoul, Korea).

\section{References}

1. Bottoms GD, Roesel OF, Rausch FD, Akins EL. Circadian variation in plasma cortisol and corticosterone in pigs and mares. Am J Vet Res. 1972, 33, 785-790.

2. Cassar G, Amezcua R, Tenbergen R, Friendship RM. Preoperative ketoprofen administration to piglets undergoing castration does not affect subsequent growth performance. Can Vet J 2014, 55, 1250-1252.

3. Garcia A, Sutherland M, Pirner G, Picinin G, May M, Backus B, McGlone J. Impact of providing feed and/or water on performance, physiology, and behavior of weaned pigs during a 32-h transport. Animals (Basel) 2016, 6, 31 .

4. Im KS, Jung HJ, Kim JB, Lee JM, Park HJ, Joo CH, Moon DE. The antinociceptive effect of acetaminophen in a rat model of neuropathic pain. Kaohsiung J Med Sci 2012, 28, 251-258.

5. Jamal SM, Belsham GJ. Foot-and-mouth disease: past, present and future. Vet Res 2013, 44, 116.

6. Keita A, Pagot E, Prunier A, Guidarini C. Pre-emptive meloxicam for postoperative analgesia in piglets undergoing surgical castration. Vet Anaesth Analg 2010, 37, 367-374.

7. Lee HS, Lee NH, Seo MG, Ko YJ, Kim B, Lee JB, Kim JS, Park S, Shin YK. Serological responses after vaccination of growing pigs with foot-and-mouth disease trivalent (type O, A and Asia1) vaccine. Vet Microbiol 2013, 164, 239-245.

8. Lee JA, Jung BG, Jung M, Kim TH, Yoo HS, Lee BJ. Dietary germanium biotite supplementation enhances the induction of antibody responses to foot-and-mouth disease virus vaccine in pigs. J Vet Sci 2014, 15, 443-447.

9. Lee JH, Kang IJ, Kim AR, Noh YS, Chung HC, Park BK. Increased humoral antibody response of foot-and-mouth disease virus vaccine in growing pigs pre-treated with poly-ãglutamic acid. J Vet Sci 2016, 17, 253-256.

10. Langhoff R, Zöls S, Barz A, Palzer A, Ritzmann M, Heinritzi K. [Investigation about the use of analgesics for the reduction of castration-induced pain in suckling piglets]. Berl Munch Tierarztl Wochenschr 2009, 122, 325-332. German.

11. Rodrigues MC, Cooke RF, Marques RS, Arispe SA, Keisler DH, Bohnert DW. Effects of oral meloxicam 
administration to beef cattle receiving lipopolysaccharide administration or vaccination against respiratory pathogens. $\mathrm{J}$ Anim Sci 2015, 93, 5018-5027.

12. Telang PS. Vitamin $\mathrm{C}$ in dermatology. Indian Dermatol Online J 2013, 4, 143-146.

13. Van Der Staay FJ, De Groot J, Van Reenen CG, HovingBolink AH, Schuurman T, Schmidt BH. Effects of Butafosfan on salivary cortisol and behavioral response to social stress in piglets. J Vet Pharmacol Ther 2007, 30, 410-416.

14. Vetvicka V, Oliveira C. $\beta(1-3)(1-6)$-D-glucans modulate immune status in pigs: potential importance for efficiency of commercial farming. Ann Transl Med 2014, 2, 16.

15. Yeruham I, Yadin H, Haymovich M, Perl S. Adverse reactions to FMD vaccine. Vet Dermatol 2001, 12, 197-201. 